\title{
Parametrically excited nonlinear magnetic rolling pendulum for broadband energy harvesting
}

\author{
Yang Kuang and Meiling Zhu* \\ College of Engineering, Mathematics and Physical Sciences, University of Exeter, Exeter, EX4 4QF, UK
}

\begin{abstract}
Parametrically excited energy harvesters provide a valuable alternative to directly excited ones. However, linear energy harvesters subjected to parametric excitation usually suffer from narrow bandwidths. This letter proposes a parametrically excited magnetic rolling pendulum (MRP) with intentionally introduced nonlinearity for broadband energy harvesting. The MRP exhibited a first-order parametric resonance with a broadened bandwidth as the excitation acceleration is $\geq 0.2 \mathrm{~g}\left(\mathrm{~g}=9.8 \mathrm{~m} / \mathrm{s}^{2}\right)$. When excited at $0.5 \mathrm{~g}$ in experiment, the parametric resonance was observed in the frequency range between $1.2 f_{0}$ and $4.5 f_{0}$ with $f_{0}$ being the natural frequency of $4 \mathrm{~Hz}$. With a peak power output of $3.6 \mathrm{~mW}$, the MRP achieved a half-power bandwidth of $4.8 \mathrm{~Hz}$, which is $120 \%$ of its natural frequency. Simulation results suggest that the power output, the bandwidth and the resonance frequency can be manipulated to by varying the magnet dimensions and positions.
\end{abstract}

Parametrically excited systems have been recently attracted the attention of the research community as a means of vibration energy harvesting. Such systems usually have rapidly varying coefficients and can be described by a homogeneous differential equation, in contrast to directly excitation systems, which lead to inhomogeneous differential equations with constant or slowly varying coefficients ${ }^{1}$. Vibration amplitudes of a parametric resonance are not limited by the linear damping but constrained by physical limits or the nonlinearity at high amplitudes ${ }^{2}$. Therefore, a small parametric actuation may produce a large response when the excitation frequency is close to double of the natural frequency (first-order parametric resonance). This mechanical amplification effect lends parametrically excited energy harvesters (PE-EHs) high electric power outputs. PE-EHs are usually implemented by applying an excitation force orthogonal to the displacement, rather than parallel to as of a directly excited one. The most common approaches of PE-EHs are vertically driven pendulum ${ }^{2,3}$ and in-plane driven cantilevers ${ }^{4,5}$. However, most of the PE-EHs in the literature are linear or with weak nonlinearity ${ }^{2,4}$, and have a very limited power bandwidth.

In recently years, in light of the success in broadband energy harvesting by intentionally introduced nonlinearity 6 , parametrically excited nonlinear energy harvesters (PE-N-EHs) have gradually attracted attention. Aevras et al. ${ }^{7}$ theoretically predicted that a parametrically excited nonlinear Mathieu system can exhibit a frequency-response curve resembling that of a monostable Duffing energy harvester. Zaghari et $a l^{8}$ experimentally and analytically proved that increased bandwidth in parametric resonance can be obtained when the nonlinear items were properly introduced, although the nonlinearity had a negative effect on the vibration amplitude growth. Despite the established theoretic work, the implementation of PE-N-EHs is limited. Ma et al. ${ }^{9}$ observed a bandwidth up to $70 \%$ of the natural frequency on a parametrically excited pendulum-based energy harvester, which becomes nonlinear as the rotation angle is large. In this letter, we propose a magnetic rolling pendulum (MRP) with a strong nonlinearity introduced by a nonlinear magnetic force for parametrically excited energy harvesting. When parametrically excited at $0.5 \mathrm{~g}$, the MRP produces a peak power of $3.6 \mathrm{~mW}$ and a of half-power bandwidth $4.8 \mathrm{~Hz}$, which is $120 \%$ of its natural frequency. The resonance range and degree of nonlinearity can be manipulated to achieve different power levels and bandwidths to suit different vibration characteristics.

The MRP (FIG. 1 (a)) consists of two coils and four neodymium (N42) magnets magnetized along their thickness direction. The dimensions of the magnets and coils are listed in TABLE 1. Magnets B, $\mathrm{C}$ and $\mathrm{O}$ are fixed to the frame, while magnet $\mathrm{A}$ is free to move. The polarisation direction of $\mathrm{A}$ is the same as $\mathrm{B}$ and $\mathrm{C}$ but opposite to $\mathrm{O}$. As a result, $\mathrm{A}$ is attracted by $\mathrm{O}$ but repelled by $\mathrm{B}$ and C. Magnet $\mathrm{O}$ constrains the motion of A on its surface. Magnets B and C provide a nonlinear magnetic spring force, which can be adjusted by the dimensions and positions of $\mathrm{B}$ and $\mathrm{C}$. When excited, magnet A rolls on the surface of magnet $\mathrm{O}$. Two coils are placed symmetrically along the passage of magnet A. As magnet A passes by the coils, it introduces variation in the magnetic flux in the coils and thus produces electricity. The MRP can be excited horizontally and vertically. When excited horizontally, it represents a nonlinear system subjected to a direct excitation. When excited vertically, the MRP is a parametrically excited nonlinear system, which is of interest in this letter.

A schematic of the modelling of the MRP is presented in FIG. 1 (b). $F_{m t}$ is the projection of the magnetic force experienced by magnet A onto the tangent direction $(+\mathrm{t})$. An inertial force $F_{i}=m A_{0} \cos (2 \pi f t)$ appears on magnet A when a harmonic excitation with acceleration amplitude of $A_{0}$ and frequency $f$ is applied. Magnet A rolls on the surface of magnet $\mathrm{O}$ at angular speed of $\dot{\varphi}$ with respect to its centre O. Assuming magnet A rolls without slipping, $\dot{\varphi}$ leads to an angular velocity $\dot{\theta}=2 \dot{\varphi}$ with respect to $\mathrm{O}$. The MRP can be represented by the following equations:

\footnotetext{
*Email:m.zhu@exeter.ac.uk
} 


$$
\begin{gathered}
M \ddot{\theta}+c_{d} \dot{\theta}-F_{m t}-m g \sin \theta=m A_{0} \cos (2 \pi f t) \sin \theta, \\
V_{O C}=-N \dot{\theta} \cdot \frac{d \Phi_{B}}{d \theta} \\
P=\frac{1}{T} \int_{0}^{T} \frac{V_{O C}^{2}}{4 R_{i}} d t .
\end{gathered}
$$

$M=2 m R_{2}+2 I_{A} / R_{2}$ is the effective mass; $I_{A}$ is the moment of inertia of magnet A with respect to its centre; $c_{d}$ is the damping factor caused by mechanical loss and electromagnetic coupling; $V_{O C}$ is the open-circuit voltage produced in the coil with $N$ windings and average magnetic flux of $\Phi_{B}$ in each winding; $P$ is the average power generated in a time period of $T$ when a load resistance $R_{i}$ is connected to the coil. $\theta$ is limited by the position angle $\alpha=0.43 \mathrm{rad}$ to $[-1.73,1.73] \mathrm{rad}$.
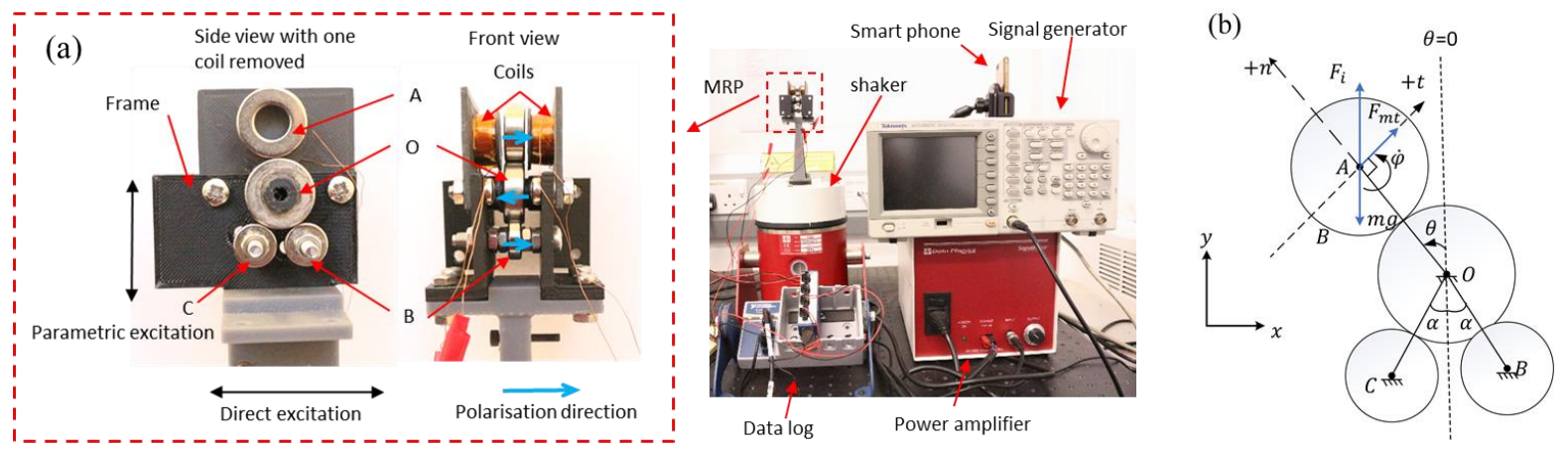

FIG. 1 (a) A prototype of the magnetic rolling pendulum sitting on the testing setup; (b) a schematic of the modelling

The magnetic force $F_{m t}$ and the magnetic flux $\Phi_{B}$ were simulated by finite element analysis in COMSOL Multiphysics® (see supplementary material) and the results are shown in FIG. 2. They can be described by Eq. ( 4 ) and ( 5 ), respectively with the parameters listed in TABLE 2.

$$
\begin{aligned}
& F_{m t}=a \theta+b \theta^{3}+c \theta^{5} \\
& \Phi_{B}=\sum_{i=0}^{7} A_{i} \cos \left(i \omega_{1} \theta\right)
\end{aligned}
$$
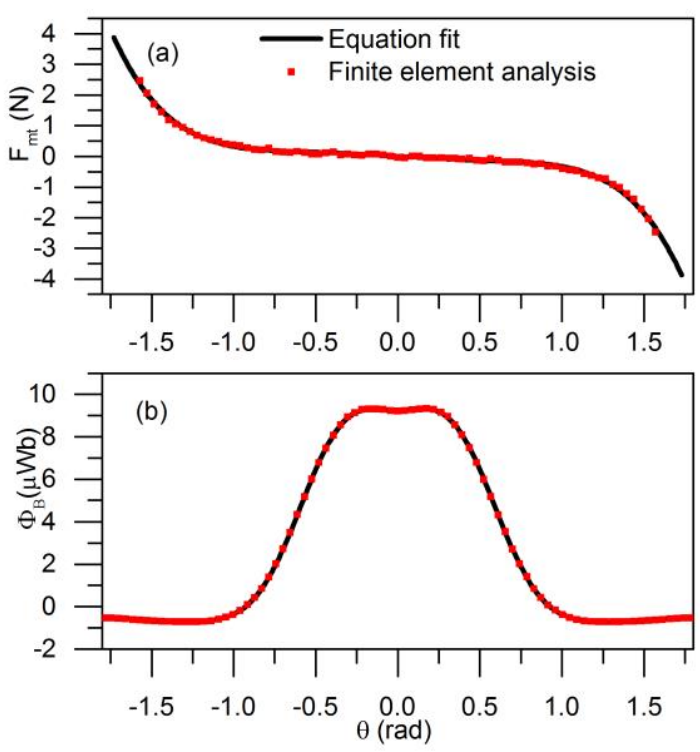

FIG. 2 Comparison of the finite element analysis results and equation fit on (a) magnetic force and (b) average magnetic flux

Substituting Eq. ( 4 ) into Eq. ( 1 ) and considering $\sin \theta \approx \theta-\frac{1}{6} \theta^{3}$, the dynamics of the MRP can be re-written as

$$
M \ddot{\theta}+c_{d} \dot{\theta}+\left(-a-m g-m A_{0} \cos (2 \pi f t)\right) \theta+\left(-b+\frac{1}{6} m g+\frac{1}{6} m A_{0} \cos (2 \pi f t)\right) \theta^{3}-c \theta^{5}=0
$$

Eq. ( 6 ) is a nonlinear Mathieu equation with time-varying coefficients in both linear and cubic nonlinear stiffness. Systems represented by equations similar to ( 6 ) have been studied experimentally and theoretically by Zaghari $e a^{8}{ }^{8}$. It was found that although the nonlinearity reduced the unbounded amplitude of a linear parametric excited system, it brought in larger power 
bandwidth, which is beneficial for energy harvesting. In this letter, both numeric simulation and experimental characterisation were performed to study the performance of the MRP.

TABLE 1 Parameters of the prototype

\begin{tabular}{llccc}
\hline \hline & Description & symbol & value & unit \\
\hline \multirow{2}{*}{ Magnets } & inner radius & $R_{1}$ & 4.75 & $\mathrm{~mm}$ \\
A and O & outer radius & $R_{2}$ & 9.55 & $\mathrm{~mm}$ \\
& thickness & $t_{A}$ & 6.4 & $\mathrm{~mm}$ \\
& mass & $m$ & 13.8 & gram \\
\hline \multirow{2}{*}{ Magnets } & inner radius & $r_{1}$ & 2 & $\mathrm{~mm}$ \\
B and C & outer radius & $r_{2}$ & 6 & $\mathrm{~mm}$ \\
& thickness & $t_{B}$ & 4 & $\mathrm{~mm}$ \\
\hline \multirow{3}{*}{ Coils } & inner radius & $R_{c 1}$ & 3 & $\mathrm{~mm}$ \\
1 and 2 & outer radius & $R_{c 2}$ & 7.9 & $\mathrm{~mm}$ \\
& thickness & $t_{c}$ & 7 & $\mathrm{~mm}$ \\
& Coil position & $d_{g a p}$ & 1.7 & $\mathrm{~mm}$ \\
\hline \hline & Coil resistance & $R_{i}$ & 10 & $\Omega$ \\
\hline
\end{tabular}

TA $\overline{\overline{B L E} 2 \text { Parameters of the magnetic force and magnetic flux }}$ flu

\begin{tabular}{cccccc}
\hline \hline parameters & values & parameters & values & parameters & values \\
\hline$a$ & -0.58 & $A_{1}$ & $2.12 \times 10^{-6}$ & $A_{5}$ & $-3.95 \times 10^{-7}$ \\
$b$ & 0.83 & $A_{2}$ & $4.20 \times 10^{-6}$ & $A_{6}$ & $-2.75 \times 10^{-7}$ \\
$c$ & -0.54 & $A_{3}$ & $1.96 \times 10^{-6}$ & $A_{7}$ & $-8.4 \times 10^{-8}$ \\
& & $A_{4}$ & $1.02 \times 10^{-7}$ & $\omega_{1}$ & 1.636 \\
\hline \hline
\end{tabular}

To test the performance, the MRP was installed on an electromagnetic shaker (V20, Data Physics), as shown in FIG. 1 (a). The shaker provides a vertical harmonic excitation at constant acceleration amplitudes range from 0.2 to $0.7 \mathrm{~g}\left(\mathrm{~g}=9.8 \mathrm{~m} / \mathrm{s}^{2}\right)$. Each coil of the MRP was connected to a $10 \Omega$ load resistor, which matches the internal resistance of the coils. The voltages across the load resistors were measured to calculate the average power generation. The camera of a smart phone (iPhone 6S) was used to record the motion of magnet A at 240 frames per second. When taking the video, one coil was removed to fully expose magnet A to the camera. The video footage was processed by Tracker 5.0 (Open Source Physics) to extract the displacement of magnet A. The process to extract the displacement from the video footage is described in the supplementary material.

In the numerical simulation, Eq. (6) was solved by an ordinary differential equation solver (ODE23) in Matlab with excitation frequency varying from 4 to $20 \mathrm{~Hz}$. At each excitation frequency, $\theta$ and $\dot{\theta}$ were solved for 50 seconds to allow magnet A to achieve a steady state. $\theta$ and $\dot{\theta}$ in the last 10 seconds of each frequency were input to Eq. (2-3) to calculate the steady-state voltage and power output. Depending on the initial disturbances, the MRP may (1) produce a high-amplitude steady-state response or (2) be trapped at the low-amplitude state. In this study, the MRP was tested and simulated in an upward frequency sweep to study its highamplitude response and its potential for energy harvesting. The initial conditions required to produce the high-amplitude response need further investigation and thus are not discussed in this letter.

FIG. 3 (a) shows the measured and simulated electric power output of the MRP in an upward frequency sweep when $A_{0}=0.3 \mathrm{~g}$. The power-frequency curve resembles that of a monostable nonlinear energy harvester ${ }^{10}$ - the response curve is bended to the right and resonance is shifted to higher frequency range due to the strong hardening nonlinearity, giving rise to an increased power bandwidth. The resonance of the MRP observed is the first-order parametric resonance. This can be verified by the steady-state displacement of the MRP shown in (b), (c) and (d). When exited at 6, 10 and $14 \mathrm{~Hz}$, the MRP shows displacement response at 3, 5 and $7 \mathrm{~Hz}$, respectively, i.e. the displacement response frequency is always half of the excitation frequency as of the first-order parametric resonance. The main frequency of the voltage (shown in (e), (f) and (g)) is twice of the displacement, which is expected because each cycle the motion introduces two cycles of flux variations in the coils. Good agreement between simulation and experiment is observed in voltage, displacement and electric power. The parametric resonance in experiment starts at $4.8 \mathrm{~Hz}$, which is $1.2 f_{0}\left(f_{0}=\right.$ $4 \mathrm{~Hz}$ ). The power then increases with frequency until a steep jump-down is observed at $15 \mathrm{~Hz}$. The steep jump-down is a phenomenon associated with systems with hardening nonlinearity. The parametric resonance is therefore covers a frequency range between $1.2 f_{0}$ and $3.75 f_{0}$. 

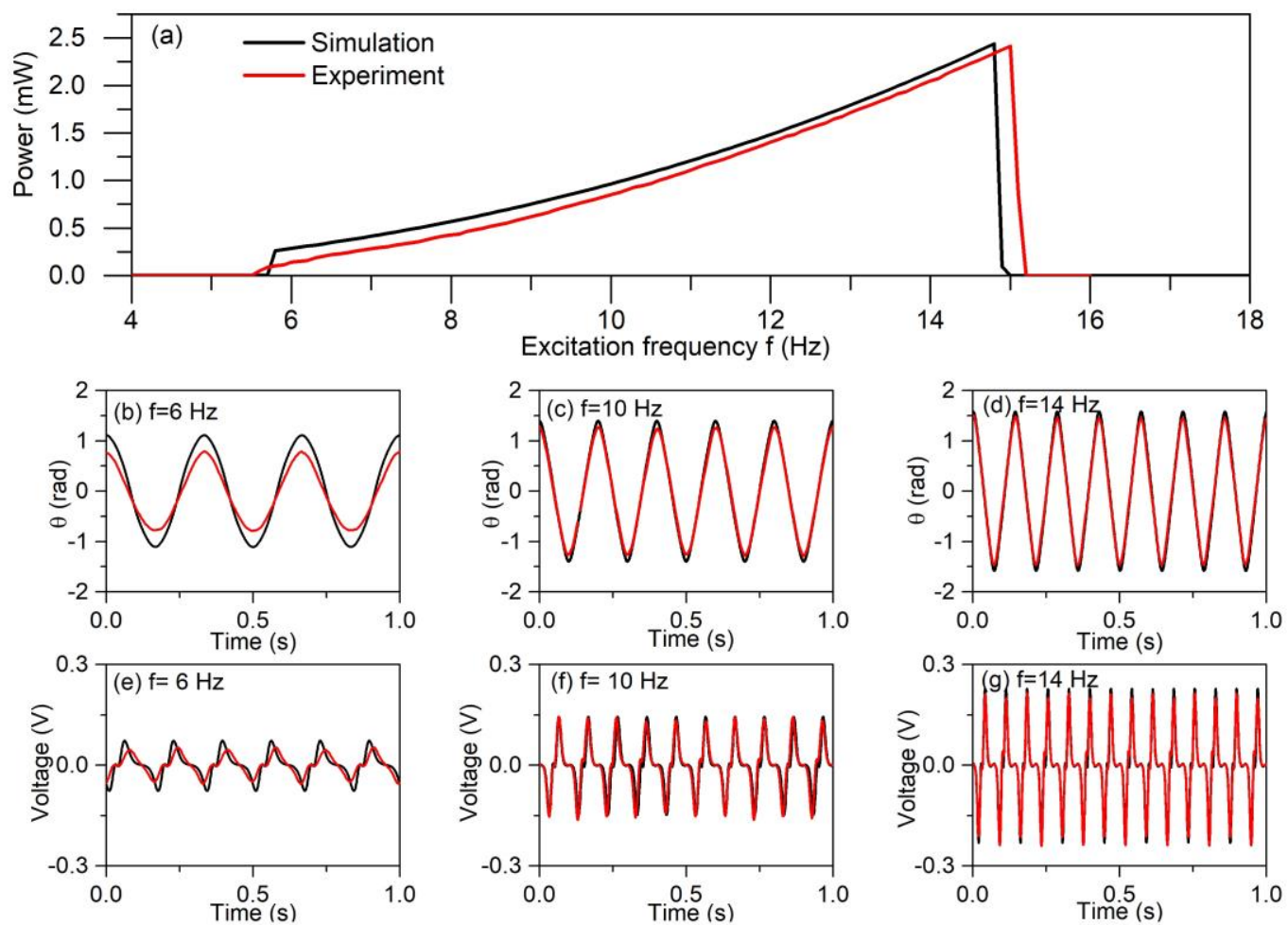

FIG. 3 Measured and simulated performance of the MRP when $A_{0}=0.3 \mathrm{~g}$ : (a) power output at different frequencies; (b), (c) and (d) steady-state displacements when $f=6,10$ and $14 \mathrm{~Hz}$, respectively; (e), (f) and (g) steady-state voltages across the $10 \Omega$ load resistor when $f=6,10$ and $14 \mathrm{~Hz}$, respectively. All time-domain results were aligned to 0 to $1 \mathrm{~s}$.

FIG. 4 (a) shows the measured electric power outputs of the MRP when different acceleration levels are applied. The parametric resonance is observed when $A_{0} \geq 0.2 \mathrm{~g}$. This means the excitation amplitude has to overcome a threshold prior to triggering parametric resonance, which is a characteristic of parametrically excited system ${ }^{4}$. As $A_{0}$ increases from 0.2 to $0.5 \mathrm{~g}$, the jump-down frequency increases from $10.1 \mathrm{~Hz}$ to $18 \mathrm{~Hz}$. As $A_{0}$ further increases, the jump-down frequency maintains around $18 \mathrm{~Hz}$. This is because the displacement of the MRP reaches the position limit: magnet A collides with magnets B and C. This can be verified by the audio observation in experiment and the displacement amplitude in simulation (FIG. 4 (b)). The displacement reaches the limit of $1.73 \mathrm{rad}$ at $18 \mathrm{~Hz}$ when $A_{0} \gg 0.5 \mathrm{~g}$. In each collision, the MRP losses a fraction of its kinetic energy and finally jumps to the low energy state. With a peak power of $3.6 \mathrm{~mW}$, the maximum parametric resonance zone is from 5 to $18 \mathrm{~Hz}$, i.e. from $1.25 f_{0}$ to $4.5 f_{0}$. The maximum half-power bandwidth measured is $4.8 \mathrm{~Hz}$, covering from 13.2 to $18 \mathrm{~Hz}$.
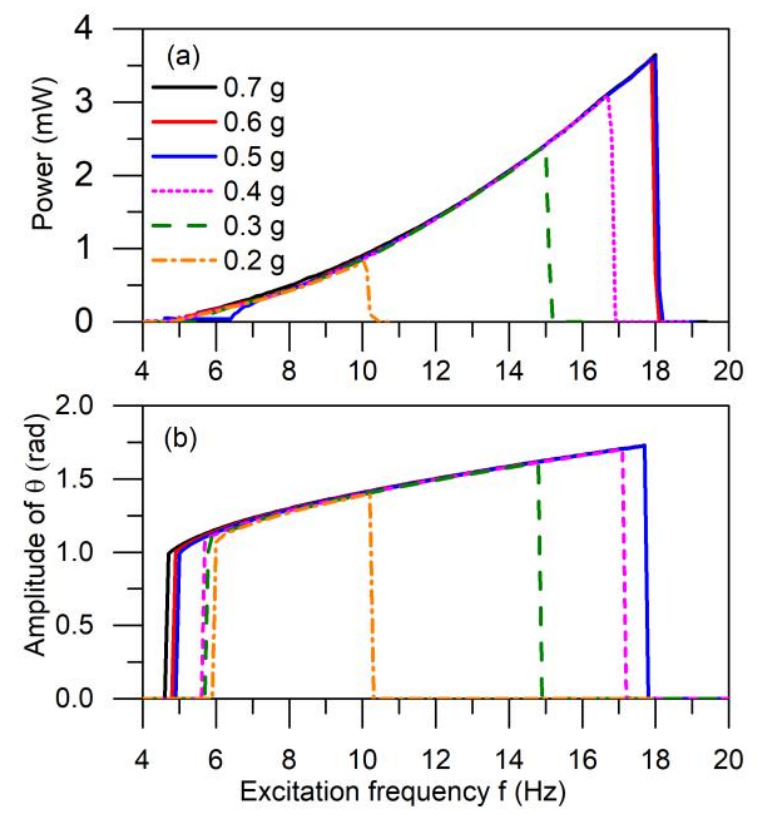

FIG. 4 (a) Measured electric power outputs and (b) simulated displacement amplitudes of the MRP subjected to different acceleration amplitudes $A_{0}$ 
It can also be observed from FIG. 4 (b) that once the parametric resonance is sustained, the displacement amplitude and the power output is independent of the excitation amplitudes, which is caused by the strong nonlinearity and was also reported in ${ }^{11}$. Although the displacement amplitude (FIG. 4 (b)) in the parametric resonance increases with frequency at a decreasing rate because of the increasing magnetic repulsive force, the power goes up progressively with the excitation frequency. This is because the power output is proportional to the square of velocity, which is proportional to frequency with the same displacement. Therefore, operating the MRP at higher frequencies might be beneficial in power generation. To verify this, we simulated the performance of the MRP with two more configurations (TABLE 3). Compared to the original MRP, both Config. 1 and 2 has a position angle increased from $25^{\circ}$ to $60^{\circ}$. This limits the displacement amplitudes to $1.1 \mathrm{rad}$ but increases the natural frequency because of the smaller distance between magnets. In addition, Config. 2 has a doubled thickness for magnets B and C, which further increases the magnetic force and the natural frequency. FIG. 5 compares the electric power output of different configurations. The applied acceleration amplitudes are the minimum level required by each configuration to achieve the maximum power output and bandwidth, i.e. when magnet $\mathrm{A}$ collides with magnets B and C. Config. 1 produced $6.1 \mathrm{~mW}$ at $27.3 \mathrm{~Hz}$ with a half-power bandwidth of $6.2 \mathrm{~Hz}$ covering from 21 to 27.3 Hz, while Config. 2 produced $9.74 \mathrm{~mW}$ at $34.3 \mathrm{~Hz}$ with a bandwidth of $7.6 \mathrm{~Hz}$ covering from 26.7 to $34.3 \mathrm{~Hz}$. Therefore, the peak power and the bandwidth of the MRP can be increased by increasing the magnetic spring force. In addition, the frequency range covered by the bandwidth can be manipulated to the frequency of interest.

TABLE 3 Parameters of different configurations of the MRP

\begin{tabular}{ccccccc}
\hline \hline Config. & $\alpha\left(^{\circ}\right)$ & $t_{B}(\mathrm{~mm})$ & $a$ & $b$ & $c$ & $f_{0}(\mathrm{~Hz})$ \\
\hline original & 25 & 4 & -0.58 & 0.83 & -0.54 & 4.0 \\
1 & 60 & 4 & -1.0 & 1.8 & -3.8 & 7.1 \\
2 & 60 & 8 & -1.6 & 1.8 & -4.9 & 9.3 \\
\hline \hline
\end{tabular}

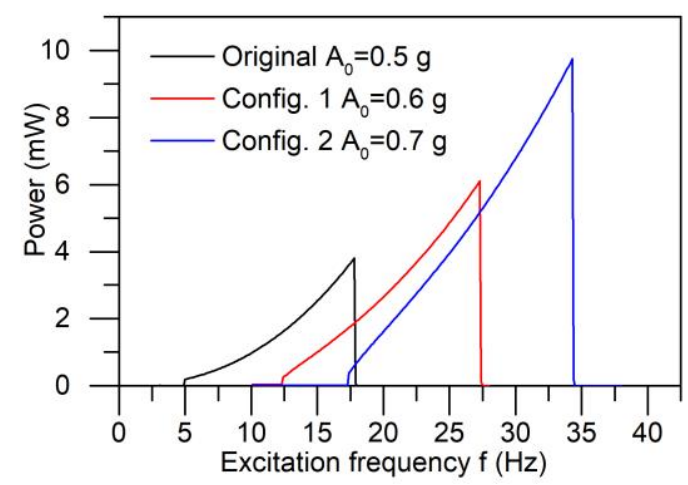

FIG. 5 Electric power outputs of the MRP with different configurations

In conclusion, we presented the experimental and simulation results for a parametrically excited magnetic rolling pendulum for broadband energy harvesting. Because of the strong nonlinearity introduced by the magnetic spring force, the parametric resonance of the MRP, which can be excited with acceleration larger than $0.2 \mathrm{~g}$, demonstrated broadband characteristics as of a monostable nonlinear oscillator. When the resonance is sustained, the power output is independent of the excitation amplitude. The present prototype exhibited a power output of $3.6 \mathrm{~mW}$ and bandwidth of $4.8 \mathrm{~Hz}$ covering from $13.2 \mathrm{to} 18 \mathrm{~Hz}$, which can be further increased by design optimisation. The frequency range covered by the bandwidth can be manipulated to suit different vibrations sources with different frequency spectrums.

See supplementary material for the finite element modelling method and the process to extract displacement from the video footage.

This research was supported by Engineering and Physical Sciences Research Council of UK (EP/K020331/1 and EP/K017950/2).

${ }^{1}$ Ali H Nayfeh and Dean T Mook, Nonlinear oscillations. (John Wiley \& Sons, 2008).

${ }^{2}$ Yu Jia, Jize Yan, Kenichi Soga, and Ashwin A Seshia, Journal of intelligent material systems and structures 25 (3), 278 (2014).

${ }^{3}$ Michał Marszal, Błażej Witkowski, Krzysztof Jankowski, Przemysław Perlikowski, and Tomasz Kapitaniak, International Journal of Non-Linear Mechanics 94, 251 (2017).

${ }^{4}$ Yu Jia, Jize Yan, Kenichi Soga, and Ashwin A Seshia, Journal of Micromechanics and Microengineering 23 (11), 114007 (2013). ${ }^{5}$ Mohammed F Daqaq, Christopher Stabler, Yousef Qaroush, and Thiago Seuaciuc-Osorio, Journal of Intelligent Material Systems and Structures 20 (5), 545 (2009).

${ }^{6}$ M. F. Daqaq, R. Masana, A. Erturk, and D. D. Quinn, Applied Mechanics Reviews 66 (4) (2014).

${ }^{7}$ Panagiotis Alevras, Stephanos Theodossiades, and Homer Rahnejat, Applied Physics Letters 110 (23), 233901 (2017).

${ }^{8}$ Bahareh Zaghari, Emiliano Rustighi, and Maryam Ghandchi Tehrani, Vibration 1 (1), 157 (2018).

${ }^{9}$ Tian-Wei Ma, Hui Zhang, and Ning-Shou Xu, Mechanical Systems and Signal Processing 28, 323 (2012).

${ }^{10}$ BP Mann and ND Sims, Journal of Sound and Vibration 319 (1-2), 515 (2009). 
${ }^{11}$ Dhiman Mallick, Andreas Amann, and Saibal Roy, Smart Materials and Structures 24 (1), 015013 (2014). 